\title{
The association of medications with sedative properties with oral health behaviour in community-dwelling older people
}

Antti Tiisanoja ${ }^{1,2}$, Anna-Maija Syrjälä ${ }^{1,2,3}$, Kaija Komulainen ${ }^{4,6}$, Sirpa Hartikainen ${ }^{5}$, Heidi Taipale ${ }^{5}$, Matti Knuuttila ${ }^{1,2}$, Pekka Ylöstalo ${ }^{1,2,6,7}$

Running title: Sedative load and oral health behaviour

Keywords: Aged, independent living, oral hygiene, dental plaque, dental care, psychotropic drugs, sedatives.

${ }^{1}$ Research Unit of Oral Health Sciences, University of Oulu

${ }^{2}$ Medical Research Center Oulu, Oulu University Hospital and University of Oulu, Oulu

${ }^{3}$ Dental training clinic, Social and Health services, City of Oulu, Oulu, Finland

${ }^{4}$ Social and Health Centre of Kuopio, Kuopio, Finland

${ }^{5}$ Kuopio Research Center of Geriatric Care, University of Eastern Finland, Kuopio, Finland

${ }^{6}$ Institute of Dentistry, University of Eastern Finland, Kuopio, Finland

${ }^{7}$ Department of Oral and Maxillofacial Diseases, Kuopio University Hospital, Kuopio, Finland

Corresponding author:

Antti Tiisanoja

Periodontology and Geriatric Dentistry

Unit of Oral Health Sciences Research

Institute of Dentistry

P.O. BOX 5281

FI-90401, University of Oulu

Finland

antti.tiisanoja@oulu.fi 


\section{Abstract}

Objectives: The objective of this study was to determine whether drugs with sedative properties are related to oral health behaviour-such as frequency of toothbrushing, using toothpaste and dental visits — and oral hygiene, measured by the number of teeth with dental plaque, among communitydwelling older people.

Methods: The study population consisted of 159 community-dwelling, dentate, non-smoking, older people from the Oral Health GeMS study (Geriatric Multidisciplinary Strategy for the Good Care of Older People study). The data were collected by interviews and clinical examinations during 20042005. Sedative properties of drugs were assessed using the Sedative Load Model. Logistic and Poisson regression models were used to estimate odds ratios/relative risks (OR/RR) and 95\% confidence intervals (CI).

Results: After adjusting for confounding factors, sedative load associated with infrequent toothbrushing (OR 1.72, CI: 0.61-4.89), toothpaste use less than twice a day (OR 3.34, CI: 1.398.12), non-regular dental visits (OR 2.28 CI: 0.91-5.30) and the number of teeth with dental plaque (RR 1.20 CI: 1.04-1.39) compared to participants without a sedative load.

Conclusions: The results of this study suggest that the use of drugs with sedative properties indicates poor oral health behaviour among older people. 


\section{$\underline{\text { Introduction }}$}

In the near future, the aging population and increasing number of dentate, older people will pose challenges to dental health services. The best way to response to these challenges is to control oral diseases by improving oral self-care and organizing appropriate dental care services. To allocate resources optimally, dental professionals need to identify those older individuals who have difficulties in performing sufficient oral self-care and who have difficulties in utilizing dental health services.

It has been shown that poor oral hygiene practices and irregular utilization of dental services are associated with several factors such as low education (1), unhealthy lifestyle (2), poor health and systemic diseases $(3,4)$, functional impairments $(5,6)$ and impaired cognitive function $(7,8)$. Whether medications or their adverse effects are associated with poor oral hygiene or irregular utilization of dental services is not known. However, drugs with sedative properties have been shown to be associated with low functional capacity (9) and impaired cognition (10, 11), which both predispose a person to poor oral hygiene practices and irregular dental visits.

Earlier studies have shown that use of drugs with sedative properties is more frequent among older people than among the general population (12) varying from 10 to 40 per cent (13-15). Drugs with sedative properties include opioids, anticonvulsants, antidepressants, antipsychotics, anxiolytics and other drugs that induce sedation as a wanted effect or an unwanted side-effect. Cumulative exposure to, and use of multiple drugs with sedative properties can be assessed by using the Sedative Load Model $(16,17)$. In this model, each drug taken by the participant is categorized into one of the four groups based on its sedative properties and the cumulative exposure is the sum of the categorical values of all the used drugs (16).

The aim of this paper was to study whether sedative load is associated with a person's oral health behaviour, such as toothbrushing frequency, using toothpaste, regularity of dental visits and amount 
of dental plaque among community-dwelling older people. Our hypothesis was that older people who use drugs with sedative properties are at risk for poor oral health behaviour due to the adverse effects of these drugs.

\section{Materials and Methods}

In this paper, we used data from the population-based Oral Health GeMS study, which was part of the larger Geriatric Multidisciplinary Strategy for the Good Care of the Elderly (GeMS) study. The original study population consisted of 1000 randomly selected subjects ( $\geq 75$ years old on the first of November in 2003) living in the city of Kuopio, who were randomized into an intervention group $(n=500)$ and a control group $(n=500)$. Comprehensive geriatric assessments and oral examinations were done to the participants belonging to the intervention group at the baseline of GeMS study, prior to the interventions (354 people out of 500 participated). We further restricted our study population to include community-dwelling, non-smoking, dentate people (at least one clinically visible tooth or dental root); the final sample included 159 participants (112 women and 47 men, mean age 79.3 SD 3.66).

Written informed consent was acquired from the participants or from their relatives or caregivers. The study protocol was approved by the ethics committee of Kuopio University Hospital and the University of Kuopio. For more detailed information about the GeMS and Oral Health GeMS studies we recommend the papers published by Lampela et al. (18) and Tikkanen et al. (19) or Komulainen et al. (20) and Tiisanoja et al. (21).

A comprehensive geriatric assessment, including interviews and clinical examinations, was done by a multidisciplinary team. The team consisted of two trained nurses, two trained physiotherapists and two physicians specializing in geriatrics. If the participant was unable to answer the questions due to his/her cognitive or other impairment, a close relative or a caregiver provided the needed information. In situations where the participant was unable to visit the local municipal health centre, 
the interviewer accompanied by a physician visited his/her home to conduct the interview and clinical examination. The participants' self-reported drug use and diagnoses were verified using prescription forms and drug packages, which the participants were asked to bring to the interview and from the medical records of local municipal health centres, home nursing services, local hospitals, and Kuopio University Hospital.

An oral examination of the participants who belonged to the intervention group of the GeMS study was done during the years 2004-2005. Two dentists interviewed the participants about their oral health and behaviour and conducted clinical oral examinations in the primary care setting of the Social and Health Centre of Kuopio or the participant's home. The dentists examined the first seven participants together as for training and calibration. Since the examination was time-consuming (one hour) and caused strain to the participants, there were no repeated examinations.

\section{Outcome variables}

In this paper we used the frequency of toothbrushing, use of toothpaste, regularity of dental visits and number of teeth with dental plaque as outcome variables. Information about oral health behaviour was obtained by the dentist during the interview. Dental plaque was measured from the buccal and palatal surfaces of all teeth, based on a visual examination after light drying with an air syringe during the clinical oral examination.

Toothbrushing was classified as brushing at least twice a day vs. less frequent. Use of toothpaste was classified as using at least twice a day vs. less frequent. Dental visits were classified as regular (annually or less frequent) vs. symptom-based or never.

\section{Explanatory variable}

Sedative load (SL) was calculated for each participant by using the Sedative Load Model (16). The model was updated in 2009 to include drugs brought to the market after the development of the original model (17). Each drug used by the participant was designated into one of four groups based 
on the sedative properties of the drug. The first group consisted of first line sedative drugs (e.g. anxiolytics, hypnotics, conventional antipsychotics, tricyclic antidepressants) and the second group consisted of drugs with sedation as a significant side-effect and drugs with a sedating component (e.g. SSRIs, atypical antipsychotics, antiepileptics). The third and fourth groups included drugs with sedation as a potential but rare adverse reaction (e.g. second-generation antihistamines) and all other drugs with no known sedative properties.

A sedative rating was assigned for each group of drugs based on their sedative properties. Drugs in group one had a numeric rating of 2 and in group two the rating was 1. Drugs in groups three and four were given a rating of 0 . The participant's sedative load is the sum of all the sedative ratings of the used drugs. Participant's sedative load was classified into two categories: with no sedative load $(n=115)$ and with sedative load $(n=44)$. More detailed information about sedative load in the GeMS study population has been provided earlier (17). Both categorized and continuous sedative load variables were used in the analyses.

\section{Other variables}

The participant's functional capability was determined by using the Lawton Instrumental Activities of Daily Living (IADL) scale (22). The scale is based on a questionnaire that includes eight domains. These domains are ability to use a telephone, purchase groceries, prepare food, do laundry, cook, handle medication and take care of personal finances and household management. The IADL score was classified into two groups: 0-6 vs. 7-8.

Co-morbidities were quantified by using a modified version of the Functional Co-morbidity Index (FCI) (23), which was developed to assess physical function in older people. The FCI included the following medical diagnoses: arthritis (rheumatoid arthritis and other connective tissue disorders), osteoporosis, asthma/chronic obstructive pulmonary disease, coronary artery disease, congestive heart failure, myocardial infarction, Parkinson's disease, stroke, diabetes mellitus, depressive 
symptoms, visual impairment, hearing impairment, and obesity (body mass index > 30). Each diagnosis included in the FCI was given a numeric value of 1 and they were summed together. Information about diagnoses were obtained from the participants themselves during the comprehensive geriatric assessments, medical records from primary health care or Kuopio University Hospital or data obtained from the Finnish Special Reimbursement Registers maintained by the Social Insurance Institution of Finland.

The Mini-Mental State Examination (MMSE) (24) was used to assess the participant's cognitive function. Scores less than 25 are considered to indicate cognitive impairment (25). Additional variables were age, gender, education ( $\geq 7$ years vs. less) and total number of drugs.

\section{$\underline{\text { Statistical methods }}$}

Logistic regression models were used to estimate odds ratios (OR) and their $95 \%$ confidence intervals (CI) for all binominal variables (toothbrushing, use of toothpaste and dental visit). Poisson regression model was used to estimate relative risk (RR) for number of teeth with dental plaque because of the skewed distribution of the variable. In the Poisson regression, number of teeth was used as an offset variable.

All models were adjusted for confounding factors based on literature (26). These factors included age, gender, education, FCI and total number of drugs. The statistical analyses were done using SPSS 22.0 software for Windows (27).

\section{$\underline{\text { Results }}$}

The characteristics of the study population, according to categories of sedative load, are presented in Table 1 and unadjusted odds ratios and relative risks in Table 2. The results obtained from the multivariate models are presented in Table 3.

After adjusting for confounding factors (age, gender, education, FCI and total number of drugs) 
participants with sedative load had a higher likelihood of having insufficient oral hygiene in terms of toothbrushing (OR: 1.72, CI: 0.61-4.89) and using toothpaste less than twice a day (OR: 3.34, CI: 1.39-8.12) when compared to participants without a sedative load. For continuous sedative load variable, the corresponding odds ratios were $1.18(0.80-1.74)$ and $1.39(1.01-1.91)$, respectively. Further adjustments for MMSE and IADL caused a slight attenuation of all above mentioned risk estimates (Table 3, Model 2 \& 3).

Participants, who had sedative load, had a higher likelihood of non-regular dental visits (OR: 2.28, CI: 0.91-5.30) compared to participants without a sedative load. With continuous sedative load the odds ratio was $1.34(0.97-1.84)$. When the number of teeth with dental plaque was used as the outcome variable, participants with sedative load had a higher likelihood of having teeth with dental plaque (RR: 1.20, CI: 1.04-1.39) than participants without a sedative load. For continuous sedative load RR was 1.09 (1.03-1.16). Risk estimates for both dental visits and the number of teeth with dental plaque, attenuated slightly after further adjustment for MMSE and IADL (Table 3, Model 2 \& 3).

\section{$\underline{\text { Discussion }}$}

To our knowledge, this is the first study to analyse whether sedative load is associated with oral health behaviour. The main finding of this study was that participants who used drugs with sedative properties tended to have poorer oral health behaviour and poorer oral hygiene than participants who did not have a sedative load.

One possible explanation for the findings is that use of drugs with sedative properties decreases a person's ability to organise a visit to a dental office and manual skills needed in brushing teeth. This explanation would be in line with previous studies, which have shown that sedative load is associated with low functional capacity (9), which in turn been shown to be associated with inadequate oral hygiene (6) and poor utilization of dental services (5). Besides the effect of sedative 
load on organising and manual skills, sedative drugs have an effect on mobility, balance (28) and muscle strength (29), which all have been shown earlier to be associated with decreased utilization of dental services $(3,30)$. With impaired mobility, the trip to a dental office becomes more difficult and the person is more likely to disregard regular visits and seek help only when necessary, such as when having a toothache. The role of functional capacity in the association between sedative drugs and oral health behaviour is supported by the observations that participants with sedative load had a lower functional capacity than participants without a sedative load. On the other hand, the importance of sedative load as an independent factor was supported by the observation that the adjustment for functional capacity (IADL) had only a minuscule effect on risk estimates (Table 3, Model 3).

Another mechanism that could explain why sedative load was associated with impaired oral health behaviour could be the side-effects of drugs with sedative properties, such as altered mood, decreased cognitive function or memory difficulties $(8,31-34)$. In addition to sedative drugs having an effect on cognition $(11,12,35)$, it is worth to keeping in mind that some of the drugs with sedative properties also have anticholinergic effects, which are known to be associated with cognitive impairment (36). We assumed that the relation between sedative load and poor oral health behaviour is to some extent mediated by poor cognitive function. However, against our assumption, adjustment for cognitive function (MMSE) did not have any essential attenuating effect on risk estimates (Table 3, Model 2). This observation suggests - besides the independent role of sedative load - that the association between sedative load and health behaviour is not essentially mediated or confounded by cognitive function.

The original GeMS study population was homogeneous in terms of ethnic origin and place of residence. The participants of this study were also quite homogeneous in relation to physical and mental status, as participants living in nursing homes were excluded. The homogeneity of the study population was further increased by excluding smokers from the study. These restrictions also 
meant that the study population became smaller which can be considered as a limitation. The small study population self-evidently decreases the credibility of the study.

In addition to the above mentioned restrictions, the effect of confounding was controlled by using regression models where all models were adjusted for age, gender, education, FCI and total number of drugs. Other factors associated with oral health behaviour, such as depression (37) and Parkinson's disease, were rare in these data. The first set of the regression models were not adjusted for MMSE or IADL because we assumed that these variables are at least partly mediating the association between the use of sedative drugs and oral health behaviour. The second and third sets of models were adjusted for cognitive function (Table 3, Model 2) and functional capacity (Table 3, Model 3), respectively, to explore more thoroughly their roles in the association between sedative load and oral health behaviour.

It should be kept in mind that the causal models can be complex. In this case, impaired cognition and low functional capacity could be either an antecedent to sedative load, they could mediate the sedative effects, or they could cause confounding in the relation of sedative load to oral health behaviour. How the findings of this study are interpreted depends on the underlying causal model. Nevertheless, whatever the role of cognitive function and functional capacity is, the results showed that sedative load is a fairly independent determinant of oral health behaviour.

\section{Concluding remarks}

In this study, oral health behaviour was assessed using four variables that describe different aspects of oral health behaviour and that are partly complementary to each other, which both increase the credibility of the findings of the present study. Although a direct cause-effect relation between the effects of sedative drugs on oral health behaviour is difficult to establish, it is reasonable to consider use of sedative drugs as an independent indicator for poor oral health behaviour among older people. This means that dental professionals could use the Sedative Load Model as a part of 
comprehensive risk assessment for poor oral health behaviour and thereby identify those elderly patients who require more thorough oral hygiene measures.

\section{Clinical relevance}

$\underline{\text { Scientific rationale for this study }}$

Poor oral health behaviour increases the risk for oral diseases, such as dental caries and periodontal infections. Dental professionals need tools for identifying older people at risk of poor oral health behaviour.

\section{Principal findings}

Participants, who had a sedative load, tended to be more likely to have insufficient oral health behaviour or poorer oral hygiene than participants who did not have sedative load.

\section{$\underline{\text { Practical implications }}$}

Use of drugs with sedative properties could be an indicator of poor oral health behaviour.

\section{$\underline{\text { Acknowledgements and sources of funding statements }}$}

We thank Paula Pesonen for her statistical guidance. The original GeMS study was supported by the

Social Insurance Institute and the City of Kuopio. Dr. Tiisanoja reports personal grant from Finnish Dental Association. Dr. Hartikainen reports grants outside the present study from MSD for lecture fees. Dr. Taipale reports grants from Janssen paid to the institution where she was employed.

Other authors have nothing to disclose. There are no conflicts of interest in this research. 
Table 1. Characteristics of the participants by different categories of sedative load

\begin{tabular}{|c|c|c|c|}
\hline \multirow{2}{*}{ Variables } & \multicolumn{2}{|c|}{ Sedative load } & \multirow[b]{2}{*}{$\mathrm{p}$} \\
\hline & 0 & $\geq 1$ & \\
\hline $\mathrm{N}$ & 115 & 44 & \\
\hline Age $($ mean $\pm \mathrm{SD})$ & $78.7 \pm 3.62$ & $80.5 \pm 3.54$ & $0.01^{\mathrm{a}}$ \\
\hline Gender, proportion of women, $\%$ & 64 & 86 & $0.006^{\mathrm{b}}$ \\
\hline Education $\geq 7$ years, $\%$ & 57 & 56 & $0.926^{\mathrm{b}}$ \\
\hline Number of teeth (mean \pm SD) & $15.2 \pm 8.01$ & $13.5 \pm 8.54$ & $0.268^{\mathrm{a}}$ \\
\hline $\begin{array}{l}\text { Number of teeth with periodontal pockets } \geq \\
4 \mathrm{~mm}(\text { mean } \pm \text { SD) }\end{array}$ & $2.75 \pm 3.48$ & $2.52 \pm 4.41$ & $0.771^{\mathrm{a}}$ \\
\hline Number of carious teeth (mean \pm SD) & $0.89 \pm 1.36$ & $1.95 \pm 3.18$ & $0.036^{\mathrm{a}}$ \\
\hline $\begin{array}{l}\text { Number of teeth with dental plaque (mean } \pm \\
\text { SD) }\end{array}$ & $7.65 \pm 7.00$ & $7.89 \pm 7.43$ & $0.857^{\mathrm{a}}$ \\
\hline Toothbrushing at least twice a day, $\%$ & 84 & 82 & $0.700^{\mathrm{b}}$ \\
\hline Interdental cleaning at least once a day, $\%$ & 18 & 14 & $0.487^{b}$ \\
\hline Use of toothpaste at least twice a day, \% & 52 & 39 & $0.126^{\mathrm{b}}$ \\
\hline Regular dental visits, $\%$ & 60 & 52 & $0.377^{\mathrm{b}}$ \\
\hline Functional Comorbidity Index $($ mean \pm SD) & $2.1 \pm 1.58$ & $2.68 \pm 1.68$ & $0.049^{\mathrm{a}}$ \\
\hline $\operatorname{MMSE}($ mean $\pm \mathrm{SD})$ & $27.4 \pm 3.84$ & $25.9 \pm 3.83$ & $0.027^{\mathrm{a}}$ \\
\hline IADL $0-6, \%$ & 17 & 39 & $0.005^{\mathrm{b}}$ \\
\hline Total number of drugs (mean \pm SD) & $5.07 \pm 3.30$ & $8.3 \pm 4.15$ & $0.001^{\mathrm{a}}$ \\
\hline
\end{tabular}

MMSE: Mini-Mental State Examination, IADL: Instrumental Activities of Daily Living. p-values: ${ }^{a}$ t-test, ${ }^{b}$ Chi-square test 
Table 2. Factors related to toothbrushing, use of toothpaste, dental visits and dental plaque

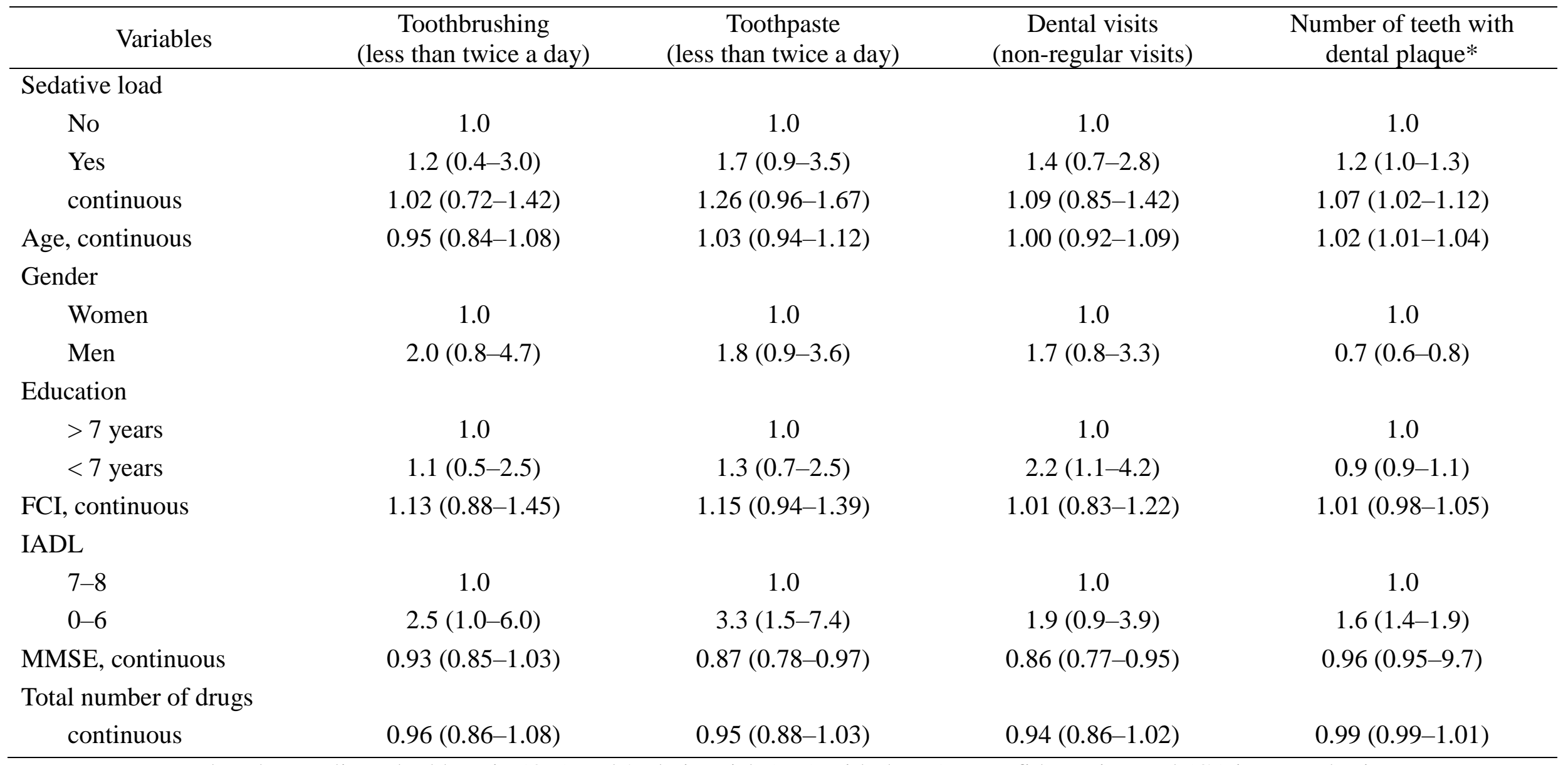

Data are presented as the unadjusted odds ratio (OR) and *relative risk (RR) with the $95 \%$ confidence interval (CI) in parenthesis.

FCI: Functional Comorbidity Index, IADL: Instrumental Activities of Daily Living, MMSE: Mini-Mental State Examination, 
Table 3. Relation between sedative load and toothbrushing, use of toothpaste and dental visits and the number of teeth with dental plaque

\begin{tabular}{|c|c|c|c|}
\hline Sedative load & $\begin{array}{c}\text { Toothbrushing } \\
\text { (less than twice a } \\
\text { day) }\end{array}$ & $\begin{array}{c}\text { Toothpaste } \\
\text { (less than twice a } \\
\text { day) }\end{array}$ & $\begin{array}{c}\text { Dental visits } \\
\text { Number of teeth } \\
\text { (non-regular visits) }\end{array}$ \\
\hline
\end{tabular}

Model 1

$\begin{array}{ccccc}\text { No } & 1.0 & 1.0 & 1.0 & 1.0 \\ \text { Yes } & 1.72(0.61-4.89) & 3.34(1.39-8.12) & 2.28(0.91-5.30) & 1.20(1.04-1.39) \\ & & & & \\ \text { continuous } & 1.16(0.78-1.71) & 1.72(1.19-2.48) & 1.34(0.97-1.84) & 1.08(1.02-1.14)\end{array}$

Model 2

No

1.0

1.0

1.0

1.0

Yes

$1.52(0.52-4.45)$

$2.87(1.16-7.16)$

$1.85(0.77-4.48)$

$1.08(0.93-1.25)$

continuous

$1.11(0.74-1.67)$

$1.62(1.12-2.34)$

$1.26(0.91-1.74)$

$1.04(0.98-1.09)$

Model 3

$\begin{array}{ccccc}\text { No } & 1.0 & 1.0 & 1.0 & 1.0 \\ \text { Yes } & 1.69(0.56-5.12) & 3.02(1.19-7.61) & 2.16(0.89-5.26) & 1.10(0.95-1.28) \\ & & & & \\ \text { continuous } & 1.12(0.73-1.69) & 1.65(1.12-2.44) & 1.30(0.93-1.83) & 1.04(0.98-1.10)\end{array}$

Data are presented as odds ratios and *relative risk (Poisson regression model, offset variable number of teeth) with the $95 \%$ confidence interval in parenthesis.

The model 1 was adjusted for age, gender, education, Functional Comorbidity Index and total number of drugs.

The model 2 was further adjusted with Mini-Mental State Examination and the model 3 with Instrumental Activities of Daily Living. 


\section{$\underline{\text { References }}$}

1 Brothwell DJ, Jay M, Schönwetter DJ. Dental service utilization by independently dwelling older adults in Manitoba, Canada. J Can Dent Assoc 2008; 74: 161-161f.

2 Harada S, Akhter R, Kurita K et al. Relationships between lifestyle and dental health behaviours in a rural population in Japan. Community Dent Oral Epidemiol 2005; 33: 17-24.

3 Kiyak HA, Reichmuth M. Barriers to and enablers of older adults' use of dental services. J Dent Educ 2005; 69: 975-986.

4 Koletsi-Kounari H, Tzavara C, Tountas Y. Health related lifestyle behaviours, sociodemographic characteristics and use of dental health services in Greek adults. Community Dent Health 2011; 28: 47-52.

5 Dolan TA, Peek CW, Stuck AE, Beck JC. Functional health and dental service use among older adults. J Gerontol A Biol Sci Med Sci 1998; 53: M413-8.

6 Komulainen K, Ylöstalo P, Syrjälä AM et al. Associations of instrumental activities of daily living and handgrip strength with oral self-care among home-dwelling elderly $75+$. Gerodontology 2012; 29: e135-142.

7 Wu B, Plassman BL, Liang J, Wei L. Cognitive function and dental care utilization among community-dwelling older adults. Am J Public Health 2007; 97: 2216-2221.

8 Moriya S, Tei K, Yamazaki Y et al. Relationships between higher-level functional capacity and dental health behaviors in community-dwelling older adults. Gerodontology 2011; 30: 133140.

9 Taipale HT, Bell JS, Gnjidic D, Sulkava R, Hartikainen S. Muscle strength and sedative load in community-dwelling people aged 75 years and older: a population-based study. J Gerontol A Biol Sci Med Sci 2011; 66: 1384-1392.

10 Shash D, Kurth T, Bertrand M et al. Benzodiazepine, psychotropic medication, and dementia: A population-based cohort study. Alzheimers Dement 2015; doi: 10.1016/j.jalz.2015.10.006. [Epub ahead of print].

11 Desplenter F, Lavikainen P, Hartikainen S, Sulkava R, Bell JS. Sedative use and incident cognitive decline among persons aged 75 years and older: a population-based longitudinal study. Int Psychogeriatr 2012; 24: 48-54.

12 Blazer D, Hybels C, Simonsick E, Hanlon JT. Sedative, hypnotic, and antianxiety medication use in an aging cohort over ten years: a racial comparison. J Am Geriatr Soc 2000; 48: 10731079.

13 Beck CA, Williams JV, Wang JL et al. Psychotropic medication use in Canada. Can J Psychiatry 2005; 50: 605-613. 
14 Hollingworth SA, Siskind DJ. Anxiolytic, hypnotic and sedative medication use in Australia. Pharmacoepidemiol Drug Saf 2010; 19: 280-288.

15 Linjakumpu TA, Hartikainen SA, Klaukka TJ et al. Sedative drug use in the home-dwelling elderly. Ann Pharmacother 2004; 38: 2017-2022.

16 Linjakumpu T, Hartikainen S, Klaukka T, Koponen H, Kivelä SL, Isoaho R. A model to classify the sedative load of drugs. Int J Geriatr Psychiatry 2003; 18: 542-544.

17 Taipale HT, Bell JS, Uusi-Kokko M, Lönnroos E, Sulkava R, Hartikainen S. Sedative load among community-dwelling people aged 75 years and older: a population-based study. Drugs Aging 2011; 28: 913-925.

18 Lampela P, Hartikainen S, Lavikainen P, Sulkava R, Huupponen R. Effects of medication assessment as part of a comprehensive geriatric assessment on drug use over a 1-year period: a population-based intervention study. Drugs Aging 2010; 27: 507-521.

19 Tikkanen P, Nykänen I, Lönroos E, Sipilä S, Sulkava R, Hartikainen S. Physical activity at age of 20-64 years and mobility and muscle strength in old age: a community-based study. $J$ Gerontol A Biol Sci Med Sci 2012; 67: 905-10.

20 Komulainen K, Ylöstalo R, Syrjälä AM et al. Determinants for preventive oral health care need among community-dwelling older people: a population-based study. Spec Care Dentist 2014; 34: 19-26.

21 Tiisanoja A, Syrjälä AM, Komulainen K et al. Sedative load and salivary secretion and xerostomia in community-dwelling older people. Gerodontology 2016; 33: 177-184.

22 Lawton MP, Brody EM. Assessment of older people: self-maintaining and instrumental activities of daily living. Gerontologist 1969; 9: 179-186.

23 Groll DL, To T, Bombardier C, Wright JG. The development of a comorbidity index with physical function as the outcome. J Clin Epidemiol 2005; 58: 595-602.

24 Crum R, Anthony JC, Bassett SS, Folstein MF. Population-based norms for the Mini-Mental State Examination by age and educational level. JAMA 1993; 269: 2386-2391.

25 Dahl A, Berg S, Nilsson SE. Identification of dementia in epidemiological research: a study on the usefulness of various data sources. Aging Clin Exp Res 2007; 19: 381-389.

26 Sundsli K, Söderhamn U, Espnes GA Söderhamn O. Ability for self-care in urban living older people in southern Norway. J Multidiscip Healthc 2012; 5: 85-95.

27 SPSS Inc. SPSS 22.0 for Windows. 2013;22.0.0.

28 Gnjidic D, Le Couteur DG, Hilmer SN et al. Sedative load and functional outcomes in community-dwelling older Australian men: the CHAMP study. Fundam Clin Pharmacol 2014; 28: $10-19$. 
29 Taipale HT, Bell JS, Gnjidic D, Sulkava R, Hartikainen S. Sedative load among communitydwelling people aged 75 years or older: association with balance and mobility. J Clin Psychopharmacol 2012; 32: 218-224.

30 Hoad-Reddick G, Grant AA, Griffiths CS. Knowledge of dental services provided: investigations in an elderly population. Community Dent Oral Epidemiol 1987; 15: 137-140.

31 Niesten D, van Mourik K, van der Sanden W. The impact of frailty on oral care behavior of older people: a qualitative study. BMC Oral Health. 2013; 13: 61. doi:10.1186/1472-6831-1361.

32 Vermeeren A. Residual effects of hypnotics: epidemiology and clinical implications. CNS Drugs 2004; 18: 297-328.

33 Chen X, Clark JJ, Naorungroj S. Cognitive impairment, oral self-care function and dental caries severity in community-dwelling older adults. Gerodontology 2015: doi: 10.1111/ger.12061. [Epub ahead of print]

34 Naorungroj S, Slade GD, Beck JD et al. Cognitive decline and oral health in middle-aged adults in the ARIC study. J Dent Res. 2013; 92: 795-801.

35 Glass J, Lanctôt KL, Herrmann N, Sproule BA, Busto UE. Sedative hypnotics in older people with insomnia: meta-analysis of risks and benefits. BMJ 2005; 331:1169.

36 Fox C, Richardson K, Maidment ID et al. Anticholinergic medication use and cognitive impairment in the older population: the medical research council cognitive function and ageing study. J Am Geriatr Soc 2011; 59: 1477-1483.

37 Anttila S, Knuuttila M, Ylöstalo P, Joukamaa M. Symptoms of depression and anxiety in relation to dental health behavior and self-percveived dental treatmen need. Eur J Oral Sci 2006; 114: 109-114. 